\title{
Zero tillage- a profitable resource conservation technology in agriculture
}

\section{Introduction}

Attaining food security for a growing population and alleviating poverty while sustaining agricultural systems under the current scenario of depleting natural resources, negative impacts of climatic variability, spiralling cost of inputs are the major challenges before Indian agriculture as well as world. In addition to these challenges, the recent stagnation of productivity in the Indo- Gangetic Plains of south Asia has also led to quest for resource conserving technologies that can save water, reduce cost of production and increase production. One of the major cropping systems of South Asia is rice-wheat, grown on 13.5million hectares on the Indo-Gangetic Plains (IGP). It has a pivotal role in the food security and livelihoods of millions of farmers and workers.

Rice-wheat systems are among the most widespread sustainability. Several studies have shown an increase in cropping system in India. The conventional systems of soil organic carbon sequestration under conservation growing rice and wheat are not very efficient in terms of agriculture in different climatic regions of the world. Water economy and the use of natural resources. Studies globally, agricultural lands have the potential to sequester have shown that timely sowing, judicious use of irrigation approximately $5500-6000 \mathrm{Mg} \mathrm{CO}-\mathrm{eq}$. yr. by 2030 . Water, appropriate management of small farms, efficient Soil carbon in its various pools within the soil, provides use of fertilizers and weed management are important structure and stability to soil. Soil organic carbon factors for maintaining the productivity of ricewheat associated with aggregates is an important reservoir of systems. Keeping in view the long-term sustainability carbon, protected from mineralization and enzymatic of the conventional rice-wheat systems, there has been degradation Soil aggregation is the process by which greater emphasis on adopting and promoting resource particles of different sizes are joined and held together by conservation technology for maintaining soil fertility and different organic and inorganic materials. There is crop productivity. The zero-tillage with wheat succeeding three size classes of soil aggregate i.e. primary particle rice is now the most widely adopted resource conserving (sand, silt and clay), micro aggregates $(53-250 \mu \mathrm{m})$ and technology in the Indo-Gangetic plains, including regions macro aggregates $(>250 \mu \mathrm{m})$ . Soil aggregation is one of Haryana and Punjab. The area under conservation the principle processes responsible for carbon tillage is estimated to be over 110million-hectares sequestration in soils and in turn, structural degradation worldwide provokes soil organic matter loss. Aggregate plays an important role in soil health i.e. movement and storage of water, soil aeration, physical protection of soil organic matter, prevention of erosion, root penetration and regulates soil microbial activity.

So, enhancing the productivity of the rice-wheat cropping system in the Indo- Gangetic Plains is of utmost importance for ensuring food security for more than $20 \%$ of the global population. Therefore, continuously, need is being felt to explore the possibilities of saving critical inputs by adopting alternative resource conservative technologies as zero tillage. The widely accepted definition of zero
Volume 6 Issue I - 2017

\author{
Sah Akhilesh \\ Birsa Agricultural University, India
}

Correspondence: Sah Akhilesh, Birsa Agricultural Unicersity, India, Email akhilesh.chiyanki@gmail.com

Received: September 20, 2017 | Published: January 30, 2017

tillage (ZT) by Philips and Young (1978) is "as system of planting (seeding) crops into untilled soil by opening a narrow slot, trench or band only of sufficient width and depth to obtain proper seed coverage. No other soil tillage is done."

ZT provide opportunities to reduce the cost of production, save water and nutrients, increase yield, improve efficient use of resources, and benefit the environment. However, there are still constraints for promotion of ZT technologies, such as lack of appropriate seeders especially for small and medium scale farmers, competition of crop residues between ZT use and livestock feeding, burning of crop residues, availability of skilled and scientific manpower. The need to develop the policy frame and strategies is urgent to promote ZT.

\section{Benefits from zero tillage}

i. Enhance production stability and yield (4-10\%)

ii. Lower production costs (Rs. 2000-3000/ha)

iii. Reduction of $\mathrm{CO}_{2}$ emission

iv. Reduction in fuel consumption

v. Lower soil erosion

vi. Increase soil quality

vii. Save water

viii. Increase biological activity

ix. Reduce health hazard by avoiding crop residue burning

$\mathrm{x}$. Enhancement of water use efficiency

xi. Reduction of the incidence of weeds such as Phalaris minor in wheat (Table 1).

\section{Constraints in adoption of ZT}

i. Lack of appropriate seeders especially for small farmers and medium scale farmers

ii. The wide spread use of crop residues for livestock feed and fuel

iii. Burning of crop residues 
iv. Lack of knowledge about the potential of ZT

v. Skilled and scientific manpower

Table I Zero Tillage adoption worldwide

\begin{tabular}{ll}
\hline Country & Area(m ha) \\
\hline USA & 26.5 \\
Brazil & 25.5 \\
Argentina & 25.5 \\
Australia & 17 \\
Indo-Gangetic Plains & 8.2 \\
China & 3.1 \\
Total & 124.8 \\
\hline
\end{tabular}

Source: FAO, 2012

\section{Conclusion}

ZT provide opportunities to reduce the cost of production, save water and nutrients, increase yield, improve efficient use of resources, and benefit the environment. However, there are still constraints for promotion of ZT technologies, such as lack of appropriate seeders especially for small and medium scale farmers, competition of crop residues between ZT use and livestock feeding, burning of crop residues, availability of skilled and scientific manpower. The need to develop the policy frame and strategies is urgent to promote ZT.

\section{Acknowledgements}

None.

\section{Conflict of interest}

The author declares no conflict of interest. 